\title{
Nine new records to inventory of continental mollusc species from Santa Catarina State, Central Southern Brazil
}

\author{
A. Ignacio Agudo-Padrón 1,", Jefferson Souza da Luz', Luís Adriano \\ Funez $^{2}$ and Ana Elisa Zermiani ${ }^{2}$
}

'Project "Avulsos Malacológicos - AM", Caixa Postal 010, CEP 88010-970, Florianópolis, Santa Catarina - SC, Brazil. *ignacioagudo@gmail.com, http://noticiasmalacologicas-am.webnode.pt

${ }^{2}$ Fundação Universidade Regional de Blumenau - FURB, Rua Antônio da Veiga, 140, Victor Konder, CEP 89012-900, Blumenau - SC, Brazil.

\begin{abstract}
A new total of nine continental gastropod forms are incorporated to the previous systematic malacological inventory of Santa Catarina's State/SC, central southern Brazil region, the species Helicina schereri Baker, 1913, Assiminea sp, Lamellaxis clavulinus (Potiez \& Michaud, 1838), Lamellaxis (Leptopeas) cf. mizius Marcus \& Marcus, 1968, Plekocheilus (Eurytus) sp, Rhinus cf. longisetus (Moricand, 1846), Simpulopsis cf. ovata Sowerby, 1822, Megalobulimus klappenbachi Leme, 1964, and Zilchogyra cleliae Weyrauch, 1965. Included in eight genera and seven families, raise the state record for 220 species and subspecies regionally known (190 gastropods - 148 terrestrial, 2 amphibian and



Keywords: Continental molluscs, Santa Catarina State, Species inventory.

Received April 22, 2014

Accepted

May 12, 2014

Released

June 30, 2014

Open Acess Full Text Article
Continuing the work of regional survey, again based on extensive literature review and the examination of samples obtained in the course of works done in the field, the present contribution incorporates the record of further nine forms to previous systematic inventories of known continental molluscs of the Santa Catarina's State/SC, geographical central portion of southern Brazil region (Agudo-Padrón, 2008, 2012; Agudo-Padrón et al., 2013a,b, 2014), including gastropod forms distributed in eight genera and seven families, to a new record of 220 species and subspecies known regionally (190 gastropods -148 terrestrial, 2 amphibian and 40 limnic/freshwater -, and 30 freshwater bivalves).

In the course of this study some "cryptic regional works" of academic nature were also rescued (Corrêa, 2010; Rosso and Darolt, 2011), as important sources for future reference of new species records, the giant native forest snail Megalobulimus sanctipauli (Ihering \& Pilsbry, 1900), by example (Gregoric et al., 2013: p.1764 - Cuadro/ Table 1). The actual full list of species so far registered in the State can be viewed at the following link $<$ http://noticias-malacologicasam.webnode.pt/news/estado-da-arteordenamento-da-malacofauna-continentalde-santa-catarina-sc-/>.

The taxonomic arrangement basically continues accompanying the original proposal of Agudo-Padrón (2008, 2012) and Agudo-Padrón et al. (2013a, 2014), based on the monographic contributions of Simone (2006) and Thomé et al. (2006). Additional information about the type material deposited in museums, known geographic distribution, corresponding synonyms and other aspects relative to the related species, can be obtained through the works of Simone 
(2006), Thomé et al. (2006), and AgudoPadrón et al. (2013b).

CLASS GASTROPODA Cuvier, 1787

SUBCLASS PROSOBRANCHIA Milne

Edwards, 1848

ORDER MESOGASTROPODA

Thiele, 1927

Family Helicinidae Férussac, 1822

Helicina schereri Baker, 1913

(Figure 1)

Blumenau Municipal District, SC, ?/?/2013 (FURB MO?); Rodeio Municipal District, SC, 17/08/2013 (FURB MO?).
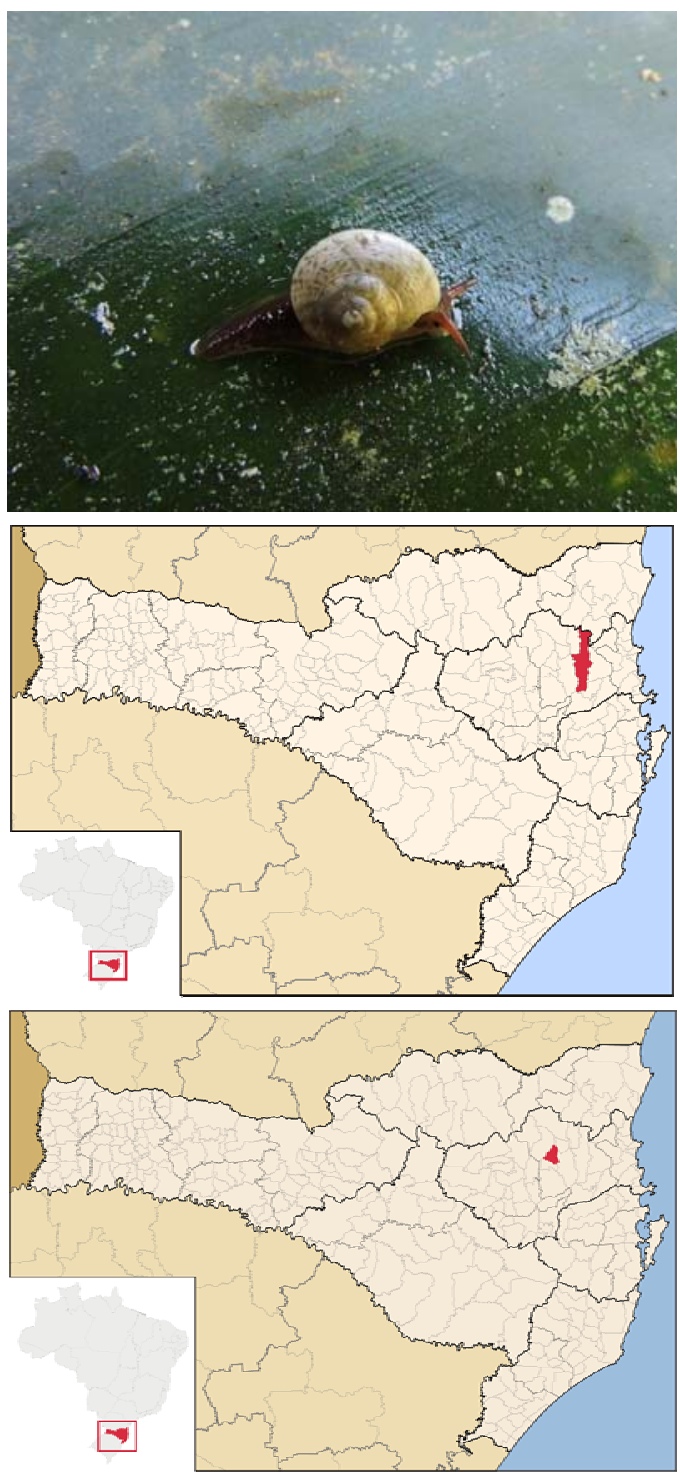

Figure 1. Helicina schereri Baker, 1913 specimen and its known distribution (Maps, red color) in Santa Catarina State/SC. Photo: Ana Elisa Zermiani.
Family Assimineidae H. Adams \& A. Adams, 1856

Assiminea sp - in determination process (Figure 2)

Rodeio Municipal District (FURB MO236, MZSP 114504); Blumenau Municipal District (FURB MO237); Gaspar Municipal District (FURB MO234); Indaial Municipal District (FURB MO190, FURB MO210). For complete report/full regional informations see Agudo et al. (2013b).
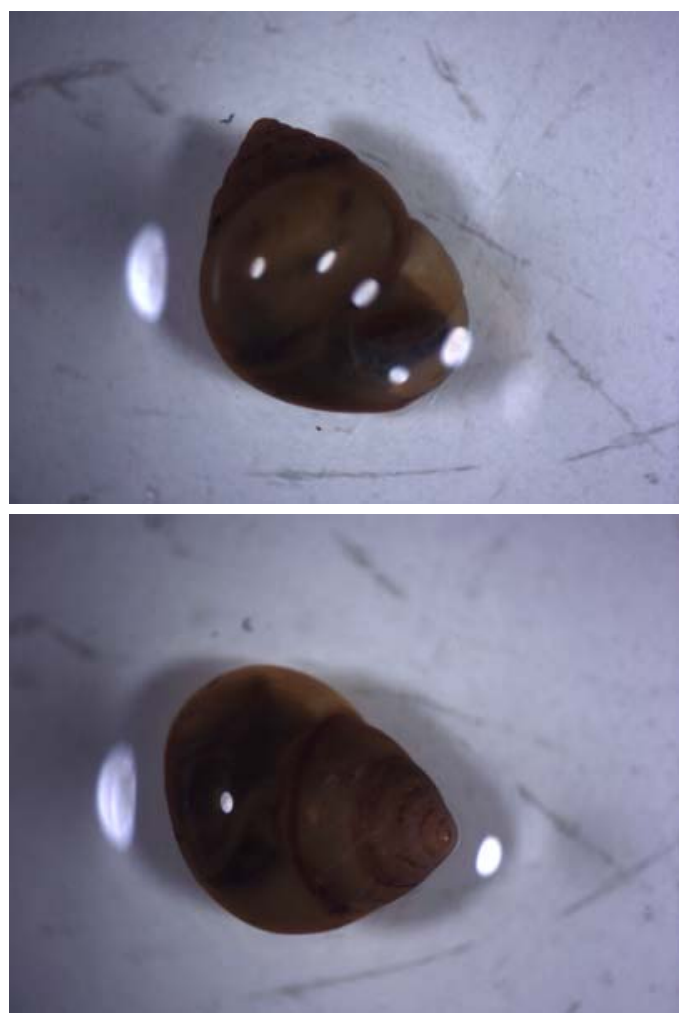

Figure 2. Assiminea sp specimen of Santa Catarina State/SC (FURB MO190). Photos: Luís Adriano Funez and Ana Elisa Zermiani.

Family Subulinidae Fischer \& Crosse, 1877

Lamellaxis clavulinus (Potiez \& Michaud, 1838) (Figure 3) Blumenau Municipal District, ?/08/2013, 1 specimen (FURB MO273). 



Figure 3. Lamellaxis clavulinus (Potiez \& Michaud, 1838) specimen and its known distribution (Map, red color) in Santa Catarina State/SC. Photo: Luís Adriano Funez.

Lamellaxis (Leptopeas) cf. mizius Marcus \& Marcus, 1968 (Figure 4)

José Milanese Municipal Ecological Park, Criciúma Municipal District, ?/?/2010 see Corrêa (2010: 24-Tabela 1 \& follows); Rodeio Municipal District, 17/08/2013, 1 specimen (FURB MO?).

Family Bulimulidae Tryon, 1896

Plekocheilus (Eurytus) sp - in determination process (Figure 5)

Itaiópolis Municipal District (High Valley of Itajaí River), 18/11/2013, 1 specimen. For a complete field information and discussion see: http://www.rabugio.org.br/ver_especie.php? id=1767; $\quad$ http://breure. wordpress.com/2013/12/02/
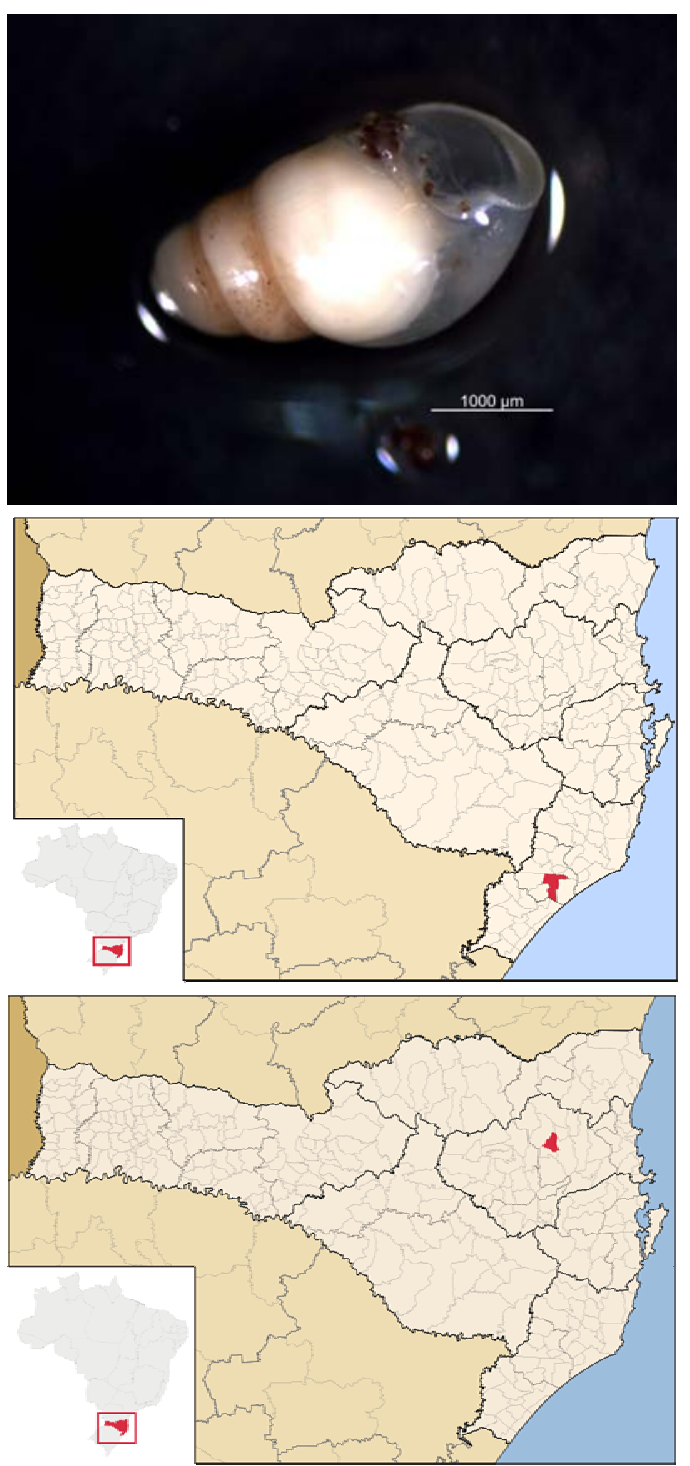

Figure 4. Lamellaxis (Leptopeas) cf. mizius Marcus \& Marcus, 1968 specimen and its known distribution (Maps, red color) in Santa Catarina State/SC. Photo: Ana Elisa Zermiani.

Rhinus cf. longisetus (Moricand, 1846) (Figure 6)

Rodeio Municipal District, 17/08/2013, 1 specimen (FURB MO?).

Family Amphibulimidae Crosse \& Fischer, 1873

Simpulopsis cf. ovata Sowerby, 1822 (Fig. 7)

Rodeio Municipal District, 17/08/2013, 1 specimen (FURB MO?). 

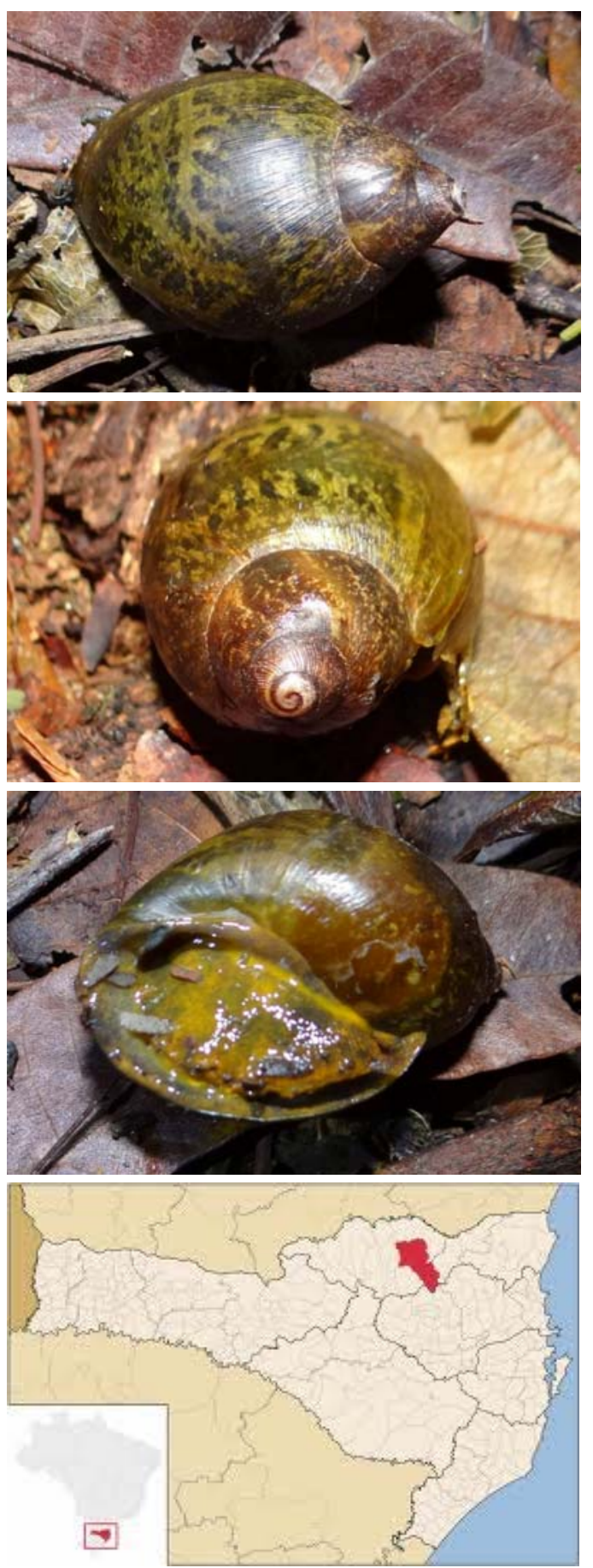

Figure 5. Plekocheilus (Eurytus) sp specimen and its known distribution (Map, red color) in Santa Catarina State/SC. Photos: Germano Woehl Jr, Instituto RãBugio, SC.
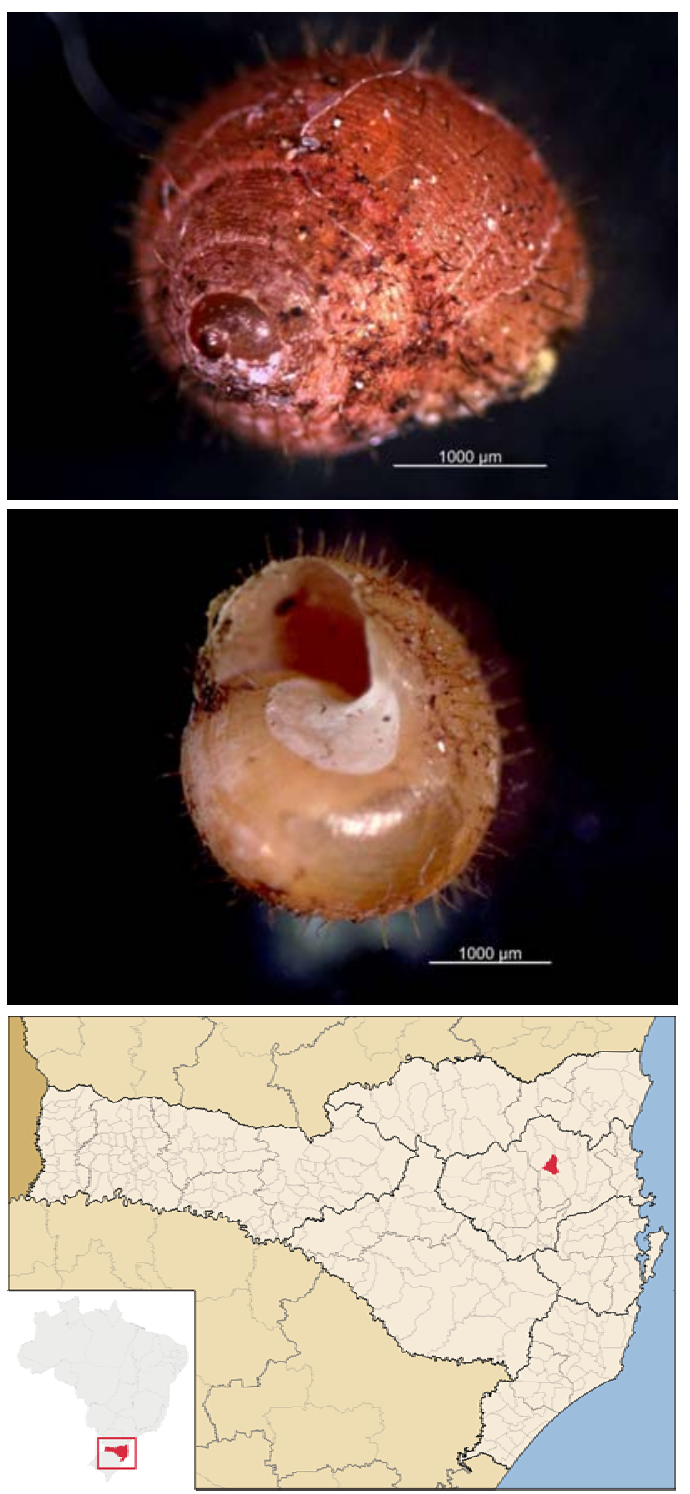

Figure 6. Rhinus cf. longisetus (Moricand, 1846) specimen and its known distribution (Map, red color) in Santa Catarina State/SC. Photos: Ana Elisa Zermiani.

Family Megalobulimidae Leme, 1973

Megalobulimus klappenbachi Leme, 1964 (Figure 8)

Garuva Municipal District see Colley (2013: p. 72). For a recognition of the species see Simone (2006, p. 212). 

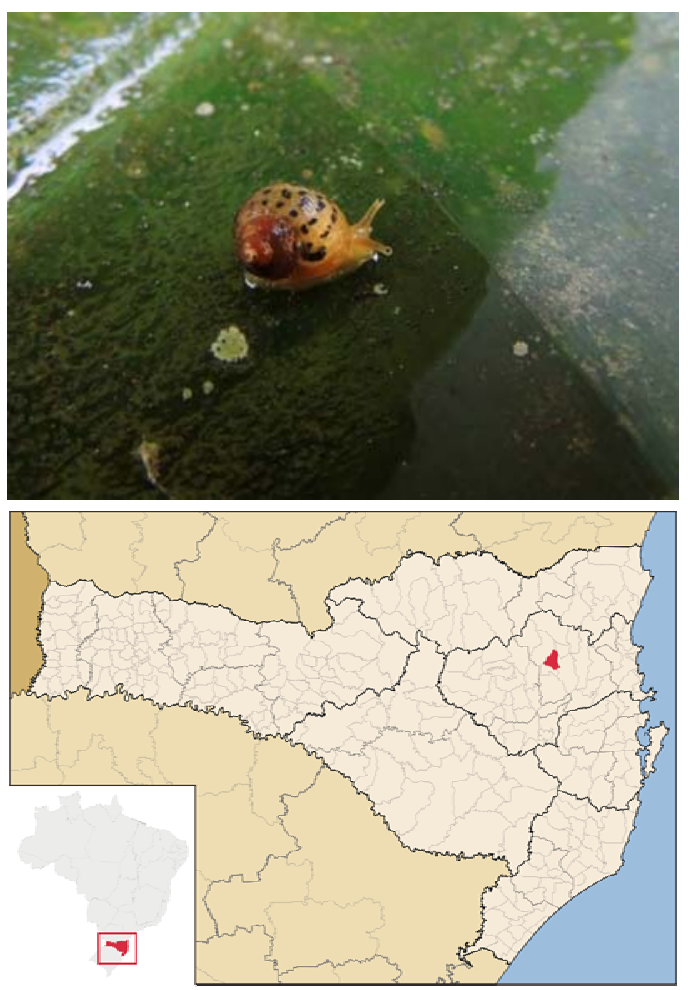

Figure 7. Simpulopsis cf. ovata Sowerby, 1822 specimen and its known distribution (Map, red color) in Santa Catarina State/SC. Photo: Ana Elisa Zermiani.

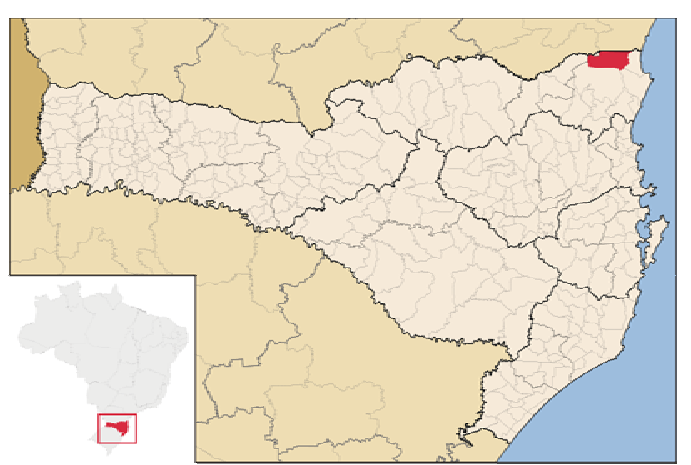

Figure 8. Known distribution (red color) in Santa Catarina State/SC of the forest snail Megalobulimus klappenbachi Leme, 1964.

Family Charopidae Hutton, 1884 Zilchogyra cleliae Weyrauch, 1965 (Figure 9)

Blumenau Municipal District, ?/08/2013, 4 specimens (FURB MO146); "Estreito" Quarter, near to "Pedro Medeiros Forest City Park", Continental Florianópolis Municipal District - see Agudo-Padrón and Luz (2014, p. 17); Joinville Municipal District (J. S. Luz et al.). For other informations, see AgudoPadrón (2008, p. 171) = Paralaoma servilis (Shuttleworth, 1852).



Figure 9. Known distribution (red color) in Santa Catarina State/SC of the micro-snail Zilchogyra cleliae Weyrauch, 1965.

Abbreviations: FURB MO Malacological Collection of Fundação Universidade Regional de Blumenau FURB, Blumenau/SC, Brazil. MZSP Malacological Collection of Museu de Zoologia da Universidade de São Paulo, São Paulo/SP, Brazil.

\section{References}

Agudo-Padrón, A. I. Listagem sistemática dos moluscos continentais ocorrentes no Estado de Santa Catarina, Brasil. Comunicaciones de la Sociedad Malacológica del Uruguay, v. 9, p. 147-179, 2008.

Agudo-Padrón, A. I. Nuevos aportes a la lista sistemática de moluscos continentales ocurrentes en el Estado de Santa Catarina, Brasil. Amici Molluscarum, v. 20, p. 35-42, 2012.

Agudo-Padrón, A. I.; Luz, J. S. Importance of preserving forest remnants in urban areas for the 
conservation of native molluscs: a study in southern Brasil. IUCN/SSC Newsletter Tentacle, n. 22, p. 16-17, 2014.

Agudo-Padrón, A. I.; Luz, J. S.; Lisboa, L. K. About four new records of continental molluscs (Gastropoda: Veronicellidae, Megalobulimidae, Vertiginidae \& Bivalvia: Hyriidae) from Santa Catarina State/SC, Central Southern Brazil. Boletín de la Asociación Argentina de Malacologia, v. 3, p. 14-19, 2013a.

Agudo-Padrón, A. I.; Funez, L. A.; Zermiani, A. E. First Record of operculated amphibian snails "Assimineidae" in Santa Catarina State/SC, Central Southern Brazil region. FMCS Newsletter Ellipsaria, v. 15, p. 34-37, 2013b.

Agudo-Padrón, A. I.; Luz, J. S.; Lisboa, L. K.; Zermiani, A. E. Additional twelve new records to inventory of continental mollusc species from Santa Catarina/SC, Central Southern Brazil. Boletín de la Asociación Argentina de Malacologia, v. 3, p. 11-20, 2014.

Colley, E. Taxonomia, Macroecologia e Ecologia de Gastropoda terrestre (Mollusca, Orthogastropoda) do Estado do Paraná, Brasil. Curitiba: Universidade Federal do Paraná, 2013. (Doctoral Thesis Zoology).
Corrêa, N. X. Comunidade de gastrópodes em áreas verdes urbanas no Município de Criciúma, SC. Criciúma: Universidade do Extremo Sul Catarinense, 2010. (TCC Bachelor Biological Sciences).

Gregoric, D. E. G.; Núñez, V.; Vogler, R. E.; Beltramino, A. A.; Rumi, A. Gasterópodos terrestres de la província de Misiones, Argentina. Revista de Biologia Tropical, v. 61, p. 1759-1768, 2013.

Rosso, P.; Darolt, S. M. Gastrópodes terrícolas na Estação Ecológica Costão da Serra, Siderópolis, Santa Catarina, Brasil. Criciúma: $1^{\circ}$ Seminário de Pesquisa, Extensão e Inovação do Instituto Federal de Santa Catarina/IF-SC - $1^{\mathrm{o}}$ SEPEI, Campus Criciúma, November 16-18, 2011. p. 25-27.

Simone, L. R. L. Land and freshwater molluscs of Brazil. São Paulo: FAPESP, 2006.

Thomé, J. W.; Gomes, S. R.; Picanço, J. B. Guia ilustrado: Os caracóis e as lesmas dos nossos bosques e jardins. Pelotas: USEB, 2006. 